\title{
Computational Complexity of Core Fragments of Modal Logics T, K4, and S4
}

\author{
Przemysław Andrzej Wałęga ${ }^{1,2}$ \\ 1 Department of Computer Science, University of Oxford, United Kingdom \\ ${ }^{2}$ Institute of Philosophy, University of Warsaw, Poland \\ p.a.walega@gmail.com
}

\begin{abstract}
We show that the satisfiability problem in core fragments of modal logics $\mathrm{T}, \mathrm{K} 4$, and $\mathrm{S} 4$ in whose languages diamond modal operators are disallowed is NL-complete. Moreover, we provide deterministic procedures for satisfiability checking. We show that the above fragments correspond to certain core fragments of linear temporal logic, hence our results imply NL-completeness of the latter.
\end{abstract}

Keywords: Modal Logic · Sub-propositional Fragments · Computational Complexity.

\section{Introduction}

Modal logics are formal systems which enable us to talk about relational structures and have a wide range of applications [3]. In order to obtain modal languages of lower computational complexity a number of methods have been introduced, e.g., restricting the nesting depth of modal operators $[8,11,12,13]$ or bounding the number of propositional variables [7]. In this paper we consider another, recently investigated, way of restricting syntax of a language which leads to sub-propositional fragments by $[6,15]$ :

- limiting formulas to the Horn, Krom, or core forms (which are analogous as in the propositional calculus $[9,10])$, denoted by "horn", "krom", and "core", respectively, in the lower index of a fragment's symbol, and

- allowing only $\square$ or only $\diamond$ operators, which is denoted by " $\square$ " and " $\diamond$ ", respectively, in the upper index of a fragment's symbol.

This method often results in fragments which have a good compromise between computational complexity and expressive power. Such fragments have been studied, e.g., in the case of linear temporal logic [1], temporal description logics [2], interval temporal logics [5,4], and, recently, normal modal logics $\mathbf{K}, \mathbf{T}, \mathbf{K} 4, \mathbf{S 4}$, and S5 $[15,6]$. Recall that $\mathbf{K}$ is the basic modal logic which semantically corresponds to the class of relational structures with an arbitrary binary relation, whereas in $\mathbf{T}, \mathbf{K} 4, \mathbf{S 4}$, and $\mathbf{S 5}$ the relation is reflexive, transitive, a preorder (i.e., reflexive and transitive), and an equivalence, respectively. In this paper we identify complexity of a logic with the computational complexity of the satisfiability problem of its formulas. It is well-known that the $\operatorname{logics} \mathbf{K}, \mathbf{T}, \mathbf{K 4}$, and 
S4 are PSPACE-complete, whereas S5 is NP-complete [11]. A Hasse diagram for fragments of these logics together with the computational complexity results is depicted in Fig. 1, where an arrow indicates a syntactical extension of a given fragment.

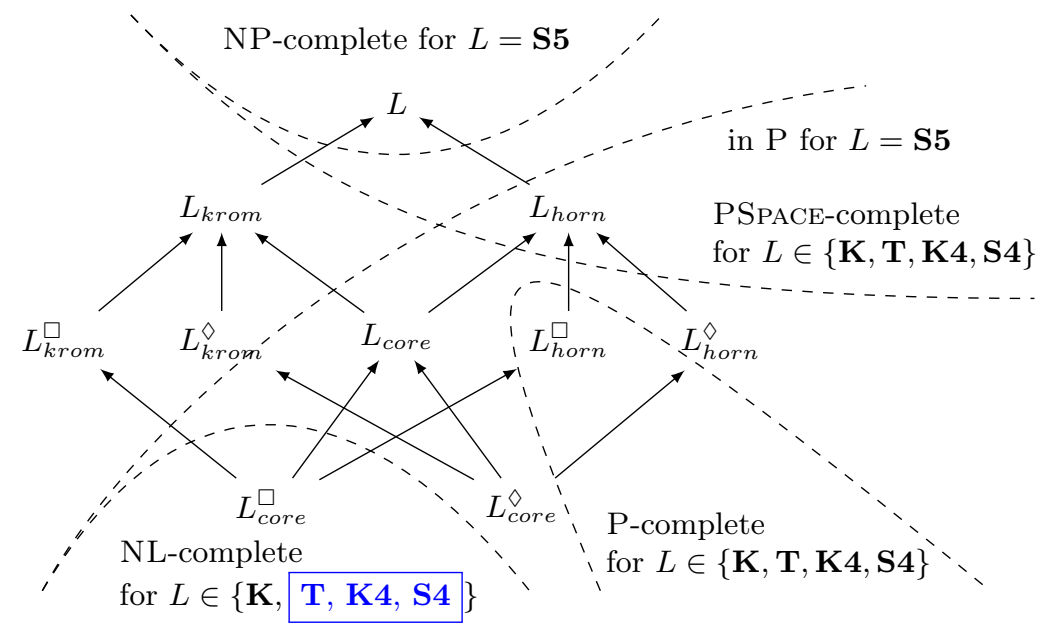

Fig. 1. Computational complexity map for sub-propositional fragments of normal modal logics, where results established in this paper are presented in a frame

In particular, it is known that $L_{\text {horn }}^{\square}$ is P-complete for $L \in\{\mathbf{K}, \mathbf{T}, \mathbf{K} 4, \mathbf{S} 4\}[6,15]$. On the other hand, its fragment $L_{\text {core }}^{\square}$ is known to be NL-complete for $L=\mathbf{K}$ but its complexity for $L \in\{\mathbf{T}, \mathbf{K} \mathbf{4}, \mathbf{S} \mathbf{4}\}$ was not known. As stated in [15], it was only known that the problem is in P (by P-completeness of $L_{\text {horn }}^{\square}[6]$ ), and NLhard (by NL-completeness of the core fragment of classical propositional calculus [14]). The core fragment is particularly interesting since it is expressive enough to represent some basic constraints used in conceptual modelling, and hence, it has a potential for practical applications [2]. On the other hand, in most of the investigated cases (e.g., in linear temporal logic [1] and interval logics [4]) tight complexity bounds for the satisfiability problem in core fragments are usually unknown.

The main result of this paper is that $L_{\text {core }}^{\square}$ for $L \in\{\mathbf{T}, \mathbf{K} 4, \mathbf{S} 4\}$ is in NL, which implies NL-completeness of these fragments. In particular, we show that given an $L_{c o r e}^{\square}$-formula $\varphi$, we can reduce the problem of checking its satisfiability in $\mathbf{S} \mathbf{4}_{\text {core }}^{\square}$ to checking if $\varphi$ is satisfiable in the minimal frame with 2 elements see Table 1. In the case of $\mathbf{T}_{\text {core }}^{\square}$ the minimal frame is of size $\operatorname{md}(\varphi)+1$, where $\operatorname{md}(\varphi)$ is the modal depth of $\varphi$, i.e., the maximum number of nested modal operators in $\varphi$. For $\mathbf{K} 4_{\text {core }}^{\square}$ there are two types of minimal frames, both of size at most $|\varphi|$, where $|\varphi|$ is the length of $\varphi$. Next, we show that for a minimal frame of $\mathbf{T}_{\text {core }}^{\square}, \mathbf{K} \mathbf{4}_{\text {core }}^{\square}$, and $\mathbf{S} \mathbf{4}_{\text {core }}^{\square}$ we can construct the minimal model based on this 
Table 1. Minimal frames for $\mathbf{K}_{\text {core }}^{\square}, \mathbf{T}_{\text {core }}^{\square}, \mathbf{K} \mathbf{4}_{\text {core }}^{\square}$, and $\mathbf{S} \mathbf{4}_{\text {core }}^{\square}$, and the corresponding LTL fragments

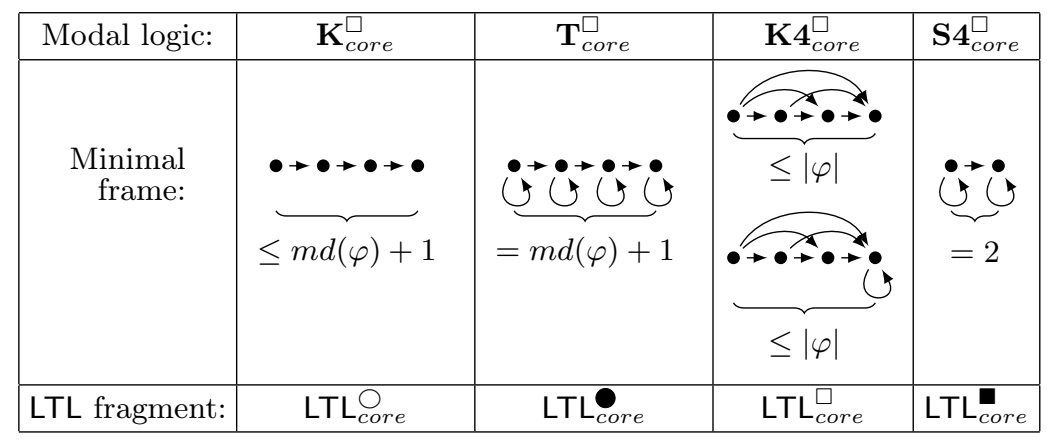

frame, such that $\varphi$ is satisfiable in the minimal frame if and only if it is satisfied in the the minimal model. As we show, the latter condition can be checked in NL, which implies that these fragments are in NL.

By the form of the minimal frames for $\mathbf{K}_{\text {core }}^{\square}, \mathbf{T}_{\text {core }}^{\square}, \mathbf{K} \mathbf{4}_{\text {core }}^{\square}$, and $\mathbf{S} \mathbf{4}_{\text {core }}^{\square}$ it follows that these logics coincide with core fragments of linear temporal logic (LTL) with temporal modal operators $\bigcirc$ 'in the next time-point', 'now and in the next time-point', $\square$ 'always in the future', and $\mathbf{\square}$ 'now and always in the future', respectively. Our results imply that the above-mentioned fragments of LTL are NL-complete over time lines of arbitrary countable length. Slightly different fragments of LTL (with clauses preceded by the universal modality) have been classified according to their computational complexity over integers in [1], where it is shown that $\mathrm{LTL}_{\text {core }}^{\square}$ with clauses preceded by universal modalities is NL-complete over integers.

The paper is organized as follows. In Section 2 we define core fragments of normal modal logics and in Section 3 we recall results on minimal frames, which were established in [15]. In Section 4 we prove that $\mathbf{T}_{\text {core }}^{\square}, \mathbf{K} \mathbf{4}_{\text {core }}^{\square}$, and $\mathbf{S} \mathbf{4}_{\text {core }}^{\square}$ are in NL, and in Section 5 we construct deterministic algorithms for the satisfiability problem in these fragments. In Section 6 we describe implications of our results for the complexity of LTL -fragments and in Section 7 we conclude the paper.

\section{Syntax and Semantics}

We start by introducing syntax and semantics of core fragments of normal modal logics. Let formulas of the core fragment with box modal operator only (i.e., without diamonds), denoted by $\mathbf{L}_{\text {core }}^{\square}$-formulas, be generated by the following abstract grammar:

$$
\begin{aligned}
& \varphi:=\lambda|\neg \lambda| \square^{s}(\neg \lambda \vee \lambda)\left|\square^{s}(\neg \lambda \vee \neg \lambda)\right| \varphi \wedge \varphi, \\
& \lambda:=\top|p| \square \lambda,
\end{aligned}
$$


where for any $s \in \mathbb{N}$ ( $\mathbb{N}$ is the set of all natural numbers including 0 ), $\square^{s} \varphi$ stands for $\underbrace{\square \ldots \square}_{s \text { times }} \varphi$ and $p \in$ PROP for PROP a countable set of propositional variables.

A Kripke frame (a frame in short) is a pair $\mathfrak{F}=(W, R)$, where $W$ is a nonempty set of worlds and $R \subseteq W \times W$ is an accessibility relation. A model based on a frame $\mathfrak{F}$ is a pair $\mathcal{M}=(\mathfrak{F}, V)$ (we will also write $\mathcal{M}=(W, R, V)$ ), where $V:$ PROP $\longrightarrow \mathcal{P}(W)$ is a valuation assigning a set of worlds to each propositional variable. The satisfaction relation $=$ for a model $\mathcal{M}=(W, R, V)$ and a world $w \in W$ is defined inductively as follows:

$$
\begin{aligned}
& \mathcal{M}, w=\top \quad \text { for all } w \in W \text {; } \\
& \mathcal{M}, w \models p \quad \text { iff } \quad w \in V(p) \text {, for all } p \in \text { PROP; } \\
& \mathcal{M}, w \models \neg \varphi \quad \text { iff } \quad \mathcal{M}, w \not \models \varphi ; \\
& \mathcal{M}, w=\varphi_{1} \wedge \varphi_{2} \quad \text { iff } \quad \mathcal{M}, w \models \varphi_{1} \text { and } \mathcal{M}, w \models \varphi_{2} \text {; } \\
& \mathcal{M}, w=\varphi_{1} \vee \varphi_{2} \quad \text { iff } \quad \mathcal{M}, w \models \varphi_{1} \text { or } \mathcal{M}, w \models \varphi_{2} \text {; } \\
& \mathcal{M}, w \models \square \varphi \quad \text { iff } \quad \mathcal{M}, w^{\prime} \models \varphi \text { for all } w^{\prime} \text { such that } R\left(w, w^{\prime}\right) \text {, }
\end{aligned}
$$

where $\varphi, \varphi_{1}$, and $\varphi_{2}$ are $\mathbf{L}_{\text {core }}^{\square}$-formulas.

For convenience we introduce an equisatisfiable grammar for $\mathbf{L}_{\text {core }}^{\square}$-formulas given by:

$$
\varphi:=\square^{s} \lambda\left|\square^{s}(p \rightarrow \lambda)\right| \square^{s}(\lambda \rightarrow p)\left|\square^{s}(p \wedge p \rightarrow \perp)\right| \varphi \wedge \varphi,
$$

where $s \in \mathbb{N}, p \in \mathrm{PROP}, \perp$ is an abbreviation for $\neg \top$, and $\varphi_{1} \rightarrow \varphi_{2}$ is an abbreviation for $\neg \varphi_{1} \vee \varphi_{2}$. We denote the set of all conjuncts in $\varphi$ by clauses $(\varphi)$ and the set of all propositional variables occurring in $\varphi$ by $\operatorname{PROP}(\varphi)$. The following result is obtained by a straight forward translation.

Proposition 1 Any formula generated by (2) can be transformed in $\mathrm{L}$ into an equisatisfiable formula generated by (1), and vice versa.

A formula $\varphi$ is $\mathbf{T}$-satisfiable if it is satisfied in some model $\mathcal{M}=(W, R, V)$ in which $R$ is reflexive. Analogously, $\varphi$ is $\mathbf{K 4}$-satisfiable if $R$ is transitive and $\varphi$ is S4-satisfiable if $R$ is transitive and reflexive.

\section{Pre-linear Models}

In this Section we will briefly recall results from [6] and [15] on minimal frames in $\mathbf{K}_{\text {core }}^{\square}, \mathbf{K} \mathbf{4}_{\text {core }}^{\square}$, and $\mathbf{S} \mathbf{4}_{\text {core }}^{\square}$, which will be useful in further Sections. Following [6], we call a frame $\mathfrak{F}=(W, R)$ a pre-linear frame if $W$ is countable (finite or infinite), i.e., $W=\left\{w_{0}, w_{1}, \ldots\right\}$ and $R=\left\{\left(w_{k-1}, w_{k}\right) \mid w_{k} \in W\right.$ and $\left.k \neq 0\right\}-$ see Fig. 2 . Moreover, for any $R \subseteq W \times W$, we denote by $R^{\circlearrowright}$ its reflexive closure, by $\vec{R}$ its transitive closure, and by $R^{*}$ its transitive and reflexive closure. If $(W, R)$ is a pre-linear frame, then $\left(W, R^{\circlearrowright}\right)$ is a pre-linear reflexive frame, $(W, \vec{R})$ is a strict linear order (asymmetric, transitive, and total relation) and $\left(W, R^{*}\right)$ a non-strict 
linear order (reflexive, antisymmetric, transitive, and total relation). A model is pre-linear, reflexive pre-linear, strict linear, or non-strict linear if it is based, respectively, on a pre-linear, reflexive pre-linear, strict linear, or non-strict linear frame. As shown in [15], pre-linear models can be used to check satisfiability of an $\mathbf{L}_{\text {core }}^{\square}$-formula, as follows:

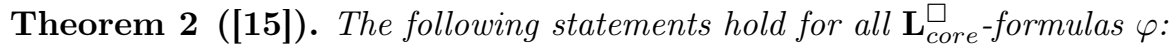

i) $\varphi$ is $\mathbf{T}$-satisfiable if and only if it is satisfiable in the root of a pre-linear reflexive model of size at most $\operatorname{md}(\varphi)+1$;

ii) $\varphi$ is K4-satisfiable if and only if it is satisfiable in the root of a model $\mathcal{M}$ of size at most $|\varphi|$ such that (i) $\mathcal{M}$ is strict linear or (ii) $\mathcal{M}$ is strict linear and additionally its last world is in accessibility relation with itself;

iii) $\varphi$ is $\mathbf{S} 4$-satisfiable if and only if it is satisfiable in the root of a non-strict linear model of size at most $|\varphi|$.

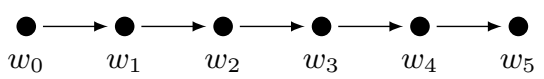

Fig. 2. A pre-linear frame $\mathfrak{F}=(W, R)$, where $W=\left\{w_{0}, \ldots, w_{5}\right\}$ and an arrow from $w_{i}$ to $w_{j}$ denotes that $R\left(w_{i}, w_{j}\right)$

\section{Computational Complexity}

By $L_{\text {core }}^{\square}$-satisfiability, for $L \in\{\mathbf{T}, \mathbf{K} 4, \mathbf{S} 4\}$, we denote the problem of checking whether a given $\mathbf{L}_{\text {core }}^{\square}$-formula is $L$-satisfiable. It is known that $L_{\text {core }}^{\square}$-satisfiability for $L \in\{\mathbf{T}, \mathbf{K 4}, \mathbf{S 4}\}$ is NL-hard and in PTime $[15,6]$ but, to the best of our knowledge, the tight complexity bounds were unknown so far. In the following Subsections we will show that each of these problems is in NL, which implies that they are all NL-complete.

\subsection{Core Fragment of $\mathrm{T}$}

The first result we will show is that $\mathbf{T}_{\text {core }}^{\square}$-satisfiability is in NL. By Theorem 2 in order to check whether an $\mathbf{L}_{c o r e}$-formula $\varphi$ is $\mathbf{T}$-satisfiable it suffices to check if $\varphi$ is satisfiable in the root of some pre-linear reflexive model of size at most $\operatorname{md}(\varphi)+1$. We will show that in order to check whether $\varphi$ is satisfiable in some pre-linear reflexive model of size $D+1$ it suffices to construct the minimal model of size $D+1$, denoted by $\mathcal{M}_{D, \varphi}^{\mathbf{T}}$, and to check whether $\varphi$ is satisfied there (Lemma 3). We show that $\mathcal{M}_{D, \varphi}^{\mathrm{T}}$ is monotonic in a sense that if a propositional variable holds in some world in $\mathcal{M}_{D, \varphi}^{\mathbf{T}}$, then this propositional variable holds in all ancestors of this world (Lemma 4). This property allows us to simplify 
rules used to construct the minimal model (Lemma 5) and to show that to check satisfiability of $\varphi$ it suffices to verify whether $\varphi$ is satisfied in the minimal model of size $m d(\varphi)+2$ (Corollary 6 ). Finally, we use the monotonicity property from Lemma 4 to show that checking whether $\varphi$ is satisfied in the minimal model of size $m d(\varphi)+2$ reduces to the reachability problem in a directed graph (Lemma 7), hence the former is in NL.

For an $\mathbf{L}_{\text {core }}^{\square}$-formula $\varphi$ and $D \in \mathbb{N}$ we introduce the minimal pre-linear reflexive model of size $D+1$ with respect to $\varphi, \mathcal{M}_{D, \varphi}^{\mathrm{T}}=(W, R, V)$, where $(W, R)$ is the pre-linear reflexive frame of size $D+1$, i.e., $W=\left\{w_{0}, \ldots, w_{D}\right\}$ and $R=\left\{\left(w_{k}, w_{k}\right) \mid k \leq D\right\} \cup\left\{\left(w_{k}, w_{k+1}\right) \mid k<D\right\}$. To define $V:$ PROP $\longrightarrow \mathcal{P}(W)$ we start by setting $V_{0}:$ PROP $\longrightarrow \mathcal{P}(W)$ such that for all $p \in$ PROP:

$$
V_{0}(p):=\left\{w_{k} \in W \mid \square^{s}\left(\square^{m} p\right) \in \operatorname{clauses}(\varphi) \text { and } k \leq s+m\right\} .
$$

For a function $f:$ PROP $\longrightarrow \mathcal{P}(W)$, let $\mathrm{cl}(f)$ be the result of non-recursive application of the below rules to $f$ :

$\left(\mathrm{cl}_{1} \mathbf{T}\right)$ If $\square^{s}\left(p_{1} \rightarrow \square^{m} p_{2}\right) \in \operatorname{clauses}(\varphi)$ and $w_{k} \in f\left(p_{1}\right)$ for some $k \leq s$, then add to $f\left(p_{2}\right)$ all $w_{l}$ such that $k \leq l \leq k+m$;

$\left(\mathrm{cl}_{2} \mathbf{T}\right)$ If $\square^{s}\left(\square^{m} p_{1} \rightarrow p_{2}\right) \in$ clauses $(\varphi)$ and for some $k \leq s$ it holds that for all $l$ such that $k \leq l \leq k+m$ we have $w_{l} \in f\left(p_{1}\right)$, then add $w_{k}$ to $f\left(p_{2}\right)$,

where $k, l \leq D$. The rules $\left(\mathrm{cl}_{1} \mathbf{T}\right)$ and $\left(\mathrm{cl}_{2} \mathbf{T}\right)$ capture semantics of formulas of the forms $\square^{s}\left(p_{1} \rightarrow \square^{m} p_{2}\right)$ and $\square^{s}\left(\square^{m} p_{1} \rightarrow p_{2}\right)$, respectively. Define the sets, obtained by subsequent applications of $\mathrm{cl}$ to $V_{0}$ as follows:

$$
\mathrm{cl}^{0}\left(V_{0}\right):=V_{0} ; \quad \mathrm{cl}^{n+1}\left(V_{0}\right):=\mathrm{cl}\left(\mathrm{cl}^{n}\left(V_{0}\right)\right) .
$$

Since $W$ and $\operatorname{PROP}(\varphi)$ are finite, there are only finitely many functions of the form $f: \operatorname{PROP}(\varphi) \longrightarrow \mathcal{P}(W)$, and so $\mathrm{cl}$ has the fixed point. We define $V$ as this fixed point. An example of $\mathcal{M}_{D, \varphi}^{\mathrm{T}}$ is presented in Fig. 3.

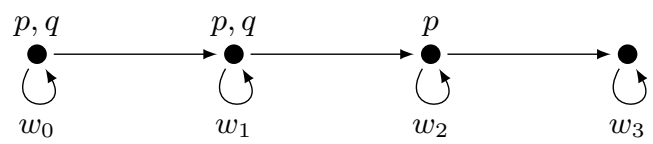

Fig. 3. The minimal model $\mathcal{M}_{D, \varphi}^{\mathbf{T}}$ for $D=3$ and $\varphi=\square^{2}(p) \wedge \square^{2}(\square p \rightarrow q)$

By the construction of the minimal model we can show that the following result holds.

Lemma 3 The following conditions are equivalent for all $\mathbf{L}_{\text {core }}^{\square}$-formulas $\varphi$ :

(1) $\varphi$ is satisfied in the root of some pre-linear reflexive model of size $D+1$;

(2) $\varphi$ is satisfied in the root of $\mathcal{M}_{D, \varphi}^{\mathrm{T}}$. 
Moreover, $\mathcal{M}_{D, \varphi}^{\mathbf{T}}$ is monotonic in the following sense.

Lemma 4 For all $p \in \mathrm{PROP}$ and $w_{k} \in W$, if $\mathcal{M}_{D, \varphi}^{\mathbf{T}}, w_{k} \models p$, then we have $\mathcal{M}_{D, \varphi}^{\mathrm{T}}, w_{l} \models p$ for all $l<k$.

This Lemma allows us to simplify construction of the minimal model by replacing $\left(\mathrm{cl}_{1} \mathbf{T}\right)$ and $\left(\mathrm{cl}_{2} \mathbf{T}\right)$ with the following rules:

$\left(\mathrm{cl}_{1} \mathbf{T}^{\prime}\right)$ If $\square^{s}\left(p_{1} \rightarrow \square^{m} p_{2}\right) \in$ clauses $(\varphi)$ and $w_{k} \in f\left(p_{1}\right)$ for some $k \leq s$, then add to $f\left(p_{2}\right)$ all $w_{l}$ such that $l \leq k+m$;

$\left(\mathrm{cl}_{2} \mathbf{T}^{\prime}\right)$ If $\square^{s}\left(\square^{m} p_{1} \rightarrow p_{2}\right) \in$ clauses $(\varphi)$ and $w_{k} \in f\left(p_{1}\right)$, then:

(i) if $k=D$, then for all $l \leq s$ add $w_{l}$ to $f\left(p_{2}\right)$;

(ii) if $k<D$, then for all $l \leq s$ such that $l \leq k-m$ add $w_{l}$ to $f\left(p_{2}\right)$.

To see that $\left(\mathrm{cl}_{2} \mathbf{T}\right)$ can be replaced with $\left(\mathrm{cl}_{2} \mathbf{T}^{\prime}\right)$ note that by Lemma 4 the model $\mathcal{M}_{D, \varphi}^{\mathbf{T}}$ is such that if $w_{k} \in V\left(p_{1}\right)$, then all its ancestors belong to $V\left(p_{1}\right)$. If $p_{1}$ is satisfied in all worlds, then a clause $\square^{s}\left(\square^{m} p_{1} \rightarrow p_{2}\right)$ forces $p_{2}$ to be satisfied in worlds $w_{0}, \ldots, w_{s}$. If $p_{1}$ is satisfied in $w_{k}$ for some $k<D$, then a clause $\square^{s}\left(\square^{m} p_{1} \rightarrow p_{2}\right)$ forces $p_{2}$ to be satisfied in $\mathcal{M}_{D, \varphi}^{\mathbf{T}}$ in worlds $w_{0}, \ldots, w_{l}$ such that $l \leq s$ and $l \leq k-m$. As we will show afterwards, the new form of rules is essential to obtain the decision procedure in NL. We can also show that the following property holds:

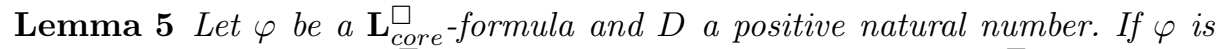
satisfied in the root of $\mathcal{M}_{D, \varphi}^{\mathrm{T}}$, then $\varphi$ is satisfied in the root of $\mathcal{M}_{D+1, \varphi}^{\mathbf{T}}$.

As a result of Lemma 5, rather than checking whether $\varphi$ is satisfied in the root of some pre-linear reflexive model of size at most $\operatorname{md}(\varphi)+1$, it suffices to check if $\varphi$ is satisfied in the root of the pre-linear reflexive model of size exactly $\operatorname{md}(\varphi)+1$.

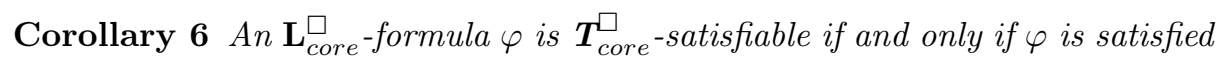
in the root of $\mathcal{M}_{m d(\varphi)+1, \varphi}^{\mathbf{T}}$.

Next, we show that checking whether $\varphi$ is satisfied in the root of $\mathcal{M}_{m d(\varphi)+1, \varphi}^{\mathbf{T}}$ reduces to the reachability problem.

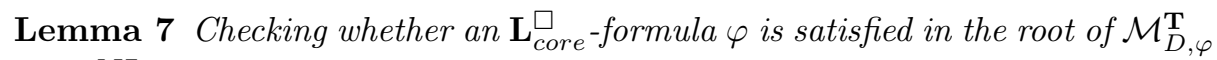
is in NL.

Proof. We use the fact that $\mathrm{NL}=\mathrm{CONL}$ [14] and introduce an NL procedure checking whether $\varphi$ is not satisfied in the root of $\mathcal{M}_{D, \varphi}^{\mathbf{T}}$.

Note that all rules in $\varphi$ which do not contain $\perp$ are satisfied in $\mathcal{M}_{D, \varphi}^{\mathrm{T}}$ by its construction. Hence, to check whether $\mathcal{M}_{D, \varphi}^{\mathrm{T}} w_{0} \not \models \varphi$ it suffices to nondeterministically guess a rule $\square^{s}\left(p_{1} \wedge p_{2} \rightarrow \perp\right)$ in $\varphi$ and then verify that $\mathcal{M}_{D, \varphi}^{\mathbf{T}}, w_{0} \not \models \square^{s}\left(p_{1} \wedge p_{2} \rightarrow \perp\right)$. If $\mathcal{M}_{D, \varphi}^{\mathbf{T}}, w_{0} \not \models \square^{s}\left(p_{1} \wedge p_{2} \rightarrow \perp\right)$, then by 
Lemma 4 we have $\mathcal{M}_{D, \varphi}^{\mathbf{T}}, w_{0} \models p_{1}$ and $\mathcal{M}_{D, \varphi}^{\mathbf{T}}, w_{0} \models p_{2}$. To check in NL whether this is the case we construct a directed graph $G=(V e r t, E)$ such that:

$$
\begin{aligned}
\text { Vert }= & \{\text { start }\} \cup\{(p, w) \mid p \in \operatorname{PROP}(\varphi) \text { and } w \in W\} \\
E= & \left\{(\text { start },(p, w)) \mid w \in V_{0}(p)\right\} \\
& \cup\left\{\left((p, w),\left(p^{\prime}, w^{\prime}\right)\right) \mid w \in f(p) \text { implies } w^{\prime} \in \mathrm{cl}(f)\left(p^{\prime}\right) \text { by }\left(\mathrm{cl}_{1} \mathbf{T}^{\prime}\right)\right\} \\
& \cup\left\{\left((p, w),\left(p^{\prime}, w^{\prime}\right)\right) \mid w \in f(p) \text { implies } w^{\prime} \in \mathrm{cl}(f)\left(p^{\prime}\right) \text { by }\left(\mathrm{cl}_{2} \mathbf{T}^{\prime}\right)\right\} .
\end{aligned}
$$

This graph (see Fig. 4), which we call an application graph, is of a polynomial size with respect to $|\varphi|$ and can be constructed in L.

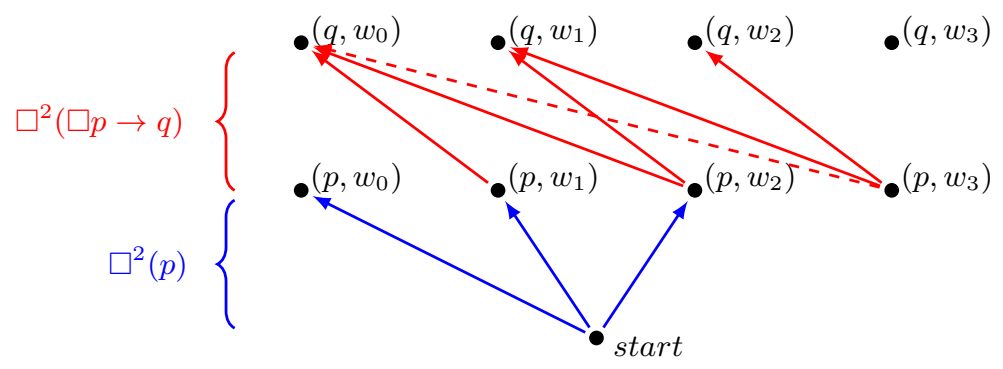

Fig. 4. An application graph for $\varphi=\square^{2}(p) \wedge \square^{2}(\square p \rightarrow q)$ and $D=3$, where curly brackets indicate clauses used to construct corresponding edges (one of the arrows is dashed only in order to make the figure more readable)

We claim that for all $p \in$ PROP and $w \in W$ we have $\mathcal{M}_{D, \varphi}^{\mathbf{T}}, w \models p$ if and only if there is a path in $G$ starting in start and ending in $(p, w)$. Indeed, assume that there is a path in $G$ from start to $(p, w)$. Edges in $E$ correspond to the construction of $V_{0}$ and applications of rules $\left(\mathrm{cl}_{1} \mathbf{T}^{\prime}\right)$ and $\left(\mathrm{cl}_{2} \mathbf{T}^{\prime}\right)$, hence $w \in V(p)$. For the other direction assume that $w \in V(p)$. Then, there is a sequence of applications of rules $\left(\mathrm{cl}_{1} \mathbf{T}^{\prime}\right)$ and $\left(\mathrm{cl}_{2} \mathbf{T}^{\prime}\right)$ to $V_{0}$ which results in adding $w$ to $V(p)$. Importantly, the rules $\left(\mathrm{cl}_{1} \mathbf{T}^{\prime}\right)$ and $\left(\mathrm{cl}_{2} \mathbf{T}^{\prime}\right)$ are linear in a sense that each of them has only one precondition, so the above-mentioned sequence of applications can be represented as a path in $G$, starting in start and ending in $(p, w)$.

We have reduced in $\mathrm{L}$ checking whether $\mathcal{M}_{D, \varphi}^{\mathbf{T}}, w \models p$ to checking if there is a path in a directed graph. The latter problem is well-known to be in NL [14], so the former is in NL as well. It follows that checking $\mathcal{M}_{D, \varphi}^{\mathbf{T}}, w_{0} \models \varphi$ is in NL.

By Corollary 6 and Lemma 7 we obtain that $\mathbf{T}_{\text {core }}^{\square}$-satisfiability is in NL, so the following result holds.

Theorem 8. $\mathbf{T}_{\text {core }}^{\square}$-satisfiability is NL-complete. 


\subsection{Core Fragment of K4}

Next, we consider $\mathbf{K} \mathbf{4}_{\text {core }}^{\square}$-satisfiability and show that it is also in NL. The proof is similar to the one for $\mathbf{T}_{c o r e}^{\square}$-satisfiability, however, there are some important differences. First, there are two types of minimal models (Lemma 9) and second, these models are monotonic but the direction of monotonicity is opposite to the direction of the monotonicity in minimal models for $\mathbf{T}$. In particular, if a propositional variable holds in a minimal model for $\mathbf{K} 4$ in some world, then it holds in all descendants of this world (Lemma 11). This property also allows us to reduce the satisfiability problem to the graph reachability problem, which implies that $\mathbf{K} \mathbf{4}_{\text {core }}^{\square}$-satisfiability is in NL (Theorem 12).

By Theorem 2 a $\mathbf{K} \mathbf{4}_{\text {core }}^{\square}$-formula $\varphi$ is satisfiable if it is satisfied in a root of a model $\mathcal{M}$ of size at most $|\varphi|$ such that (i) $\mathcal{M}$ is strict linear or (ii) $\mathcal{M}$ is strict linear and additionally its last world is in accessibility relation with itself. For a fixed $D \in \mathbb{N}$ we introduce two types of minimal strict linear models with respect to $\varphi$, namely $\mathcal{M}_{D, \varphi}^{\mathbf{K} 4(i)}$ and $\mathcal{M}_{D, \varphi}^{\mathrm{K} 4(i i)}$ which correspond to (i) and (ii), respectively. Let $\mathcal{M}_{D, \varphi}^{\mathbf{K} 4(i)}=(W, R, V)$ and $\mathcal{M}_{D, \varphi}^{\mathbf{K} 4(i i)}=\left(W, R^{\prime}, V^{\prime}\right)$ be such that $W=\left\{w_{0}, \ldots, w_{D}\right\}, \stackrel{R}{R}=\left\{\left(w_{i}, w_{j}\right) \mid i<j \leq D\right\}$, and $R^{\prime}=R \cup\left\{\left(w_{D}, w_{D}\right)\right\}$. We define $V_{0}:$ PROP $\longrightarrow \mathcal{P}(W)$ and $V_{0}^{\prime}:$ PROP $\longrightarrow \mathcal{P}(W)$ such that for all $p \in \mathrm{PROP}$ :

$$
\begin{aligned}
V_{0}(p): & =\left\{w_{k} \in W \mid \square^{s}\left(\square^{m} p\right) \in \operatorname{clauses}(\varphi) \text { and } k \geq s+m\right\} \\
& \cup\left\{w_{k} \in W \mid \square^{s}\left(\square^{m} q \rightarrow p\right) \in \operatorname{clauses}(\varphi), k \geq s, \text { and } k+m>D\right\} ; \\
V_{0}^{\prime}(p): & =\left\{w_{k} \in W \mid \square^{s}\left(\square^{m} p\right) \in \operatorname{clauses}(\varphi) \text { and } k \geq s+m\right\} \\
& \cup\left\{w_{D} \mid \square^{s}\left(\square^{m} p\right) \in \operatorname{clauses}(\varphi) \text { for all } s, m \in \mathbb{N}\right\} .
\end{aligned}
$$

For a function $f:$ PROP $\longrightarrow \mathcal{P}(W)$, let $\mathrm{cl}_{(i)}(f)$ be the result of non-recursive application of the following rules to $f$ :

$\left(\mathrm{cl}_{1} \mathbf{K} 4(i)\right)$ If $\square^{s}\left(p_{1} \rightarrow \square^{m} p_{2}\right) \in \operatorname{clauses}(\varphi)$ and $w_{k} \in f\left(p_{1}\right)$ for some $k \geq s$, then add to $f\left(p_{2}\right)$ all $w_{l}$ such that $l \geq k+m$;

$\left(\mathrm{cl}_{2} \mathbf{K} 4(i)\right)$ If $\square^{s}\left(\square^{m} p_{1} \rightarrow p_{2}\right) \in$ clauses $(\varphi)$ and for some $k \geq s$ it holds that for all $l$ with $l \geq k+m$ we have $w_{l} \in f\left(p_{1}\right)$, then add $w_{k}$ to $f\left(p_{2}\right)$,

where $k, l \leq D$. Similarly, $\mathrm{cl}_{(i i)}(f)$ is the result of non-recursive application of the following rules to $f: \mathrm{PROP} \longrightarrow \mathcal{P}(W)$ :

$\left(\mathrm{cl}_{1} \mathbf{K} 4(i i)\right)$ If $\square^{s}\left(p_{1} \rightarrow \square^{m} p_{2}\right) \in \operatorname{clauses}(\varphi)$ and $w_{k} \in f\left(p_{1}\right)$ for some $k \geq s$, then add to $f\left(p_{2}\right)$ the world $w_{D}$ and all $w_{l}$ such that $l \geq k+m$;

$\left(\mathrm{cl}_{2} \mathbf{K} 4(i i)\right)$ If $\square^{s}\left(\square^{m} p_{1} \rightarrow p_{2}\right) \in \operatorname{clauses}(\varphi), w_{D} \in f\left(p_{1}\right)$, and for some $k \geq s$ it holds that for all $l$ such that $l \geq k+m$ we have $w_{l} \in f\left(p_{1}\right)$, then add $w_{k}$ to $f\left(p_{2}\right)$,

where $k, l \leq D$. We define:

$$
\mathrm{cl}_{(x)}^{0}\left(V_{0}\right):=V_{0} ; \quad \mathrm{cl}_{(x)}^{n+1}\left(V_{0}\right):=\mathrm{cl}_{(x)}\left(\mathrm{cl}_{(x)}^{n}\left(V_{0}\right)\right) .
$$


Since $W$ and $\operatorname{PROP}(\varphi)$ are finite, there is a fixed point of $\mathrm{cl}_{(x)}$ for $x \in\{i, i i\}$. Let $V$ be the fixed point of $\mathrm{cl}_{(i)}$ and $V^{\prime}$ the fixed point of $\mathrm{cl}_{(i i)}$. The models $\mathcal{M}_{D, \varphi}^{\mathrm{K} 4(i)}$ and $\mathcal{M}_{D, \varphi}^{\mathbf{K} 4(i i)}$ satisfy the following properties.

Lemma 9 The following hold for all $\mathbf{L}_{\text {core }}^{\square}$ formulas $\varphi$ and all $D \in \mathbb{N}$ :

(1) $\mathcal{M}_{D, \varphi}^{\mathbf{K} 4(i)}, w_{0} \models \varphi$ if an only if $\varphi$ is satisfied in the root of a strict linear model of size $D+1$

(2) $\mathcal{M}_{D, \varphi}^{\mathbf{K} 4(i i)}, w_{0} \models \varphi$ if an only if $\varphi$ is satisfied in the root of a strict linear model of size $D+1$ with additional accessibility relation from the last world to itself.

Corollary 10 An $\mathbf{L}_{\text {core }}^{\square}$-formula $\varphi$ is $\mathbf{K} \mathbf{4}_{\text {core }}^{\square}$-satisfiable if an only if for some $D \leq|\varphi|-1$ we have $\mathcal{M}_{D, \varphi}^{\mathbf{K} 4(i)}, w_{0} \models \varphi$ or $\mathcal{M}_{D, \varphi}^{\mathbf{K} 4(i i)}, w_{0}=\varphi$.

By the transitivity of the accessibility relation in $\mathbf{K} \mathbf{4}$ we can show that both $\mathcal{M}_{D, \varphi}^{\mathbf{K} 4(i)}$ and $\mathcal{M}_{D, \varphi}^{\mathbf{K 4} 4(i i)}$ are monotonic in the following sense.

Lemma 11 Let $\varphi$ be an $\mathbf{L}_{\text {core }}^{\square}$ formula, $D \in \mathbb{N}, p \in \mathrm{PROP}, x \in\{i, i i\}$, and $w_{k} \in W$ such that $w_{k} \neq w_{0}$. If $\mathcal{M}_{D, \varphi}^{\mathbf{K} 4(x)}, w_{k}=p$, then $\mathcal{M}_{D, \varphi}^{\mathbf{K} 4(x)}, w_{l} \models p$ for all $w_{l} \in W$ such that $l>k$.

Therefore, each of $\left(\mathrm{cl}_{2} \mathbf{K} \mathbf{4}(i)\right)$ and $\left(\mathrm{cl}_{2} \mathbf{K} 4(i i)\right)$ can be replaced with the following rule:

$\left(\mathrm{cl}_{2} \mathbf{K} 4^{\prime}\right)$ If $\square^{s}\left(\square^{m} p_{1} \rightarrow p_{2}\right) \in \operatorname{clauses}(\varphi)$, and $w_{k} \in f\left(p_{1}\right)$ for some $w_{k} \neq w_{0}$, then for any $l \geq k-m$ with $l \geq s$ add $w_{l}$ to $f\left(p_{2}\right)$.

Theorem 12. K4 $4_{\text {core }}^{\square}$-satisfiability is NL-complete.

Proof. Fix an $\mathbf{L}_{\text {core }}^{\square}$-formula $\varphi$ and nondeterministically guess the natural number $D \leq|\varphi|-1$ and the type of a model, i.e., $x \in\{i, i i\}$. By Corollary 10 the formula $\varphi$ is $\mathbf{K} 4_{\text {core }}^{\square}$-satisfiable if and only if $\mathcal{M}_{D, \varphi}^{\mathbf{K} 4(i)}, w_{0}=\varphi$ or $\mathcal{M}_{D, \varphi}^{\mathbf{K} 4(i i)}, w_{0}=\varphi$. Both of the latter conditions reduce to reachability in the application graph which we construct in an analogous way as in the proof of Lemma 7. It follows that the whole procedure is in NL. The matching lower bound is well-known.

\subsection{Core Fragment of S4}

In this Section we will study the satisfiability problem in $\mathbf{S} \mathbf{4}_{\text {core }}^{\square}$. We will show that to check whether an $\mathbf{S} \mathbf{4}_{\text {core }}^{\square}$-formula is satisfiable in a frame of size $D+1$ it suffices to construct the minimal model $\mathcal{M}_{D, \varphi}^{\mathbf{S 4}}$ and check whether the formula holds there (Lemma 13). Then, we show that if a propositional variable holds in a non-root world in a minimal model $\mathcal{M}_{D, \varphi}^{\mathbf{S} 4}$, then it holds everywhere in this model (Lemma 14). Hence, for all $D>1$ we can construct a surjective p-morphism from $\mathcal{M}_{D, \varphi}^{\mathbf{S 4}}$ to $\mathcal{M}_{1, \varphi}^{\mathbf{S} 4}$, so $\mathbf{S} \mathbf{4}_{\text {core }}^{\square}$-satisfiability reduces to checking whether $\mathcal{M}_{1, \varphi}^{\mathbf{S} 4}, w_{0} \models \varphi$ (Corollary 15), which can be solved in NL (Theorem 16). 
For a fixed $\mathbf{L}_{c o r e}^{\square}$-formula $\varphi$, let $\mathcal{M}_{D, \varphi}^{\mathbf{S 4}}=(W, R, V)$ be the minimal non-strict linear model of size $D+1$, where $(W, R)$ is the non-strict linear frame of size $D+1$, i.e., $W=\left\{w_{0}, \ldots, w_{D}\right\}$ and $R=\left\{\left(w_{i}, w_{j}\right) \mid i \leq j \leq D\right\}$. We define $V_{0}: \mathrm{PROP} \longrightarrow \mathcal{P}(W)$ such that for all $p \in \mathrm{PROP}:$

$$
\begin{aligned}
V_{0}(p): & =\left\{w_{0} \mid \square^{0}\left(\square^{0} p\right) \in \text { clauses }(\varphi)\right\} \\
& \cup\left\{w_{0}, \ldots, w_{D} \mid \square^{s}\left(\square^{m} p\right) \in \text { clauses }(\varphi) \text { and } s+m>0\right\} .
\end{aligned}
$$

For a function $f:$ PROP $\longrightarrow \mathcal{P}(W)$, let $\operatorname{cl}(f)$ be the result of non-recursive application of the following rules to $f$ :

$\left(\mathrm{cl}_{1} \mathbf{S} 4\right)$ If $\square^{0}\left(p_{1} \rightarrow p_{2}\right) \in$ clauses $(\varphi)$ and $w_{0} \in f\left(p_{1}\right)$, then add $w_{0}$ to $f\left(p_{2}\right)$;

$\left(\mathrm{cl}_{2} \mathbf{S} \mathbf{S}\right)$ If $\square^{s}\left(p_{1} \rightarrow p_{2}\right) \in$ clauses $(\varphi)$ and $w \in f\left(p_{1}\right)$, then add $w$ to $f\left(p_{2}\right)$;

$\left(\mathrm{cl}_{3} \mathbf{S} 4\right)$ If $\square^{0}\left(p_{1} \rightarrow \square^{m} p_{2}\right) \in \operatorname{clauses}(\varphi)$ and $w_{0} \in f\left(p_{1}\right)$, then $f\left(p_{2}\right):=W$;

$\left(\mathrm{cl}_{4} \mathbf{S} 4\right)$ If $\square^{s}\left(p_{1} \rightarrow \square^{m} p_{2}\right) \in \operatorname{clauses}(\varphi)$ and $w_{k} \in f\left(p_{1}\right)$, add $w_{k}, \ldots, w_{D}$ to $f\left(p_{2}\right)$;

$\left(c_{5} \mathbf{S} 4\right)$ If $\square^{0}\left(\square^{m} p_{1} \rightarrow p_{2}\right) \in \operatorname{clauses}(\varphi)$ and $f\left(p_{1}\right)=W$, then add $w_{0}$ to $f\left(p_{2}\right)$;

$\left(\mathrm{cl}_{6} \mathbf{S} 4\right)$ If $\square^{s}\left(\square^{m} p_{1} \rightarrow p_{2}\right) \in \operatorname{clauses}(\varphi)$ and $w_{k}, \ldots, w_{D} \in f\left(p_{1}\right)$, then add $w_{k}$ to $f\left(p_{2}\right)$,

where $m, s>0$. We define the following sets, obtained by applying cl to $V_{0}$ :

$$
\mathrm{cl}^{0}\left(V_{0}\right):=V_{0} ; \quad \mathrm{cl}^{n+1}\left(V_{0}\right):=\mathrm{cl}\left(\mathrm{cl}^{n}\left(V_{0}\right)\right) .
$$

Let $V$ be the fixed point of cl. For an example of $\mathcal{M}_{D, \varphi}^{\mathrm{S} 4}$ see Fig. 5 .

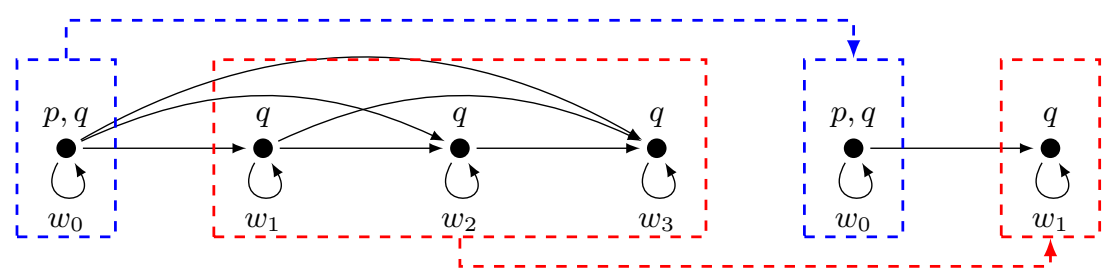

Fig. 5. The minimal models $\mathcal{M}_{3, \varphi}^{\mathbf{S} 4}$ and $\mathcal{M}_{1, \varphi}^{\mathbf{S} 4}$ for $\varphi=\square^{0}(p) \wedge \square^{2}(p \rightarrow \square q)$, where the p-morphism from $\mathcal{M}_{3, \varphi}^{\mathbf{S} 4}$ to $\mathcal{M}_{1, \varphi}^{\mathbf{S} 4}$ is indicated with dashed arrows

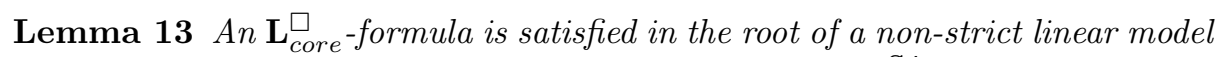
of size $D+1$ if and only if $\varphi$ is satisfied in the root of $\mathcal{M}_{D, \varphi}^{\mathbf{S 4}}$.

Furthermore, if a propositional variable is satisfied in $\mathcal{M}_{D, \varphi}^{\mathbf{S 4}}$ in some $w_{k} \neq w_{0}$, then this variable is satisfied in all worlds of $\mathcal{M}_{D, \varphi}^{\mathbf{S} 4}$.

Lemma 14 Let $\varphi$ be an $\mathbf{L}_{\text {core }}^{\square}$ formula, $D \in \mathbb{N}$, and $\mathcal{M}_{D, \varphi}^{\mathbf{S} 4}=(W, R, V)$. For all $k \geq 1$ and $p \in \mathrm{PROP}$ if $\mathcal{M}_{D, \varphi}^{\mathbf{S} 4}, w_{k} \models p$, then $\mathcal{M}_{D, \varphi}^{\mathbf{S} 4}, w \models p$ for all $w \in W$. 
As a consequence of Lemma 14 and the form of the rules $\left(\mathrm{cl}_{1} \mathbf{S} 4\right)-\left(\mathrm{cl}_{6} \mathbf{S} \mathbf{4}\right)$ we can show that for all $D>1$ there is a surjective p-morphism from $\mathcal{M}_{D, \varphi}^{\mathbf{S} 4}$ to $\mathcal{M}_{1, \varphi}^{\text {S4 }}$ (for a description of p-morphisms see [3]). This surjective p-morphism maps the root of $\mathcal{M}_{D, \varphi}^{\mathrm{S} 4}$ into the root of $\mathcal{M}_{1, \varphi}^{\mathrm{S} 4}$ and all non-root worlds of $\mathcal{M}_{D, \varphi}^{\mathrm{S} 4}$ into the non-root world of $\mathcal{M}_{1, \varphi}^{\mathbf{S 4}}$ as depicted in Fig. 5. Then, by the existence of such a p-morphism, $\mathcal{M}_{D, \varphi}^{\mathbf{S} 4}, w_{0} \models \varphi$ implies $\mathcal{M}_{1, \varphi}^{\mathbf{S} 4}, w_{0} \models \varphi$. Moreover, by the form of the rules $\left(\mathrm{cl}_{1} \mathbf{S} 4\right)-\left(\mathrm{cl}_{6} \mathbf{S} \mathbf{S}\right)$ it is easy to show that $\mathcal{M}_{0, \varphi}^{\mathbf{S 4}}, w_{0} \models \varphi$ implies $\mathcal{M}_{1, \varphi}^{\mathbf{S} 4}, w_{0}=\varphi$, hence:

Lemma 15 An $\mathbf{L}_{\text {core-formula } \varphi} \varphi$ is $\mathbf{S} \mathbf{4}_{\text {core }}^{\square}$-satisfiable if and only if it is satisfied in the root of $\mathcal{M}_{1, \varphi}^{\mathbf{S} 4}$.

It follows that to check if an $\mathbf{L}_{\text {core }}^{\square}$-formula is $\mathbf{S} \mathbf{4}_{\text {core }}^{\square}$-satisfiable it is sufficient check whether $\mathcal{M}_{1, \varphi}^{\mathbf{S} 4}, w_{0} \models \varphi$. By Lemma 14 if $D>0$, then the rules $\left(\mathrm{cl}_{5} \mathbf{S} 4\right)$ and $\left(\mathrm{Cl}_{6} \mathbf{S} \mathbf{4}\right)$ can be replaced with the following:

$\left(\mathrm{cl}_{5} \mathbf{S} 4^{\prime}\right)$ If $\square^{0}\left(\square^{m} p_{1} \rightarrow p_{2}\right) \in$ clauses $(\varphi)$ and $w_{1} \in f\left(p_{1}\right)$, then add $w_{0}$ to $f\left(p_{2}\right)$;

$\left(\mathrm{cl}_{6} \mathbf{S} 4^{\prime}\right)$ If $\square^{s}\left(\square^{m} p_{1} \rightarrow p_{2}\right) \in \operatorname{clauses}(\varphi)$ and $w_{1} \in f\left(p_{1}\right)$, then $f\left(p_{2}\right)=W$,

where $s>0$. Each of the rules $\left(\mathrm{cl}_{1} \mathbf{S} 4\right)-\left(\mathrm{cl}_{4} \mathbf{S} 4\right),\left(\mathrm{cl}_{5} \mathbf{S} 4^{\prime}\right)$, and $\left(\mathrm{cl}_{6} \mathbf{S} 4^{\prime}\right)$ has only one precondition, so we can construct an application graph for $\mathcal{M}_{1, \varphi}^{\mathbf{S} 4}$ analogously as in the proof of Lemma 7 . Hence, $\mathbf{S} \mathbf{4}_{\text {core }}^{\square}$-satisfiable reduces to reachability, so it is in NL.

Theorem 16. $\mathbf{S} \mathbf{4}_{\text {core }}^{\square}$-satisfiability is NL-complete.

\section{$5 \quad$ Algorithms}

In the previous Section we have showed that the satisfiability problems for $\mathbf{T}_{\text {core }}^{\square}$, $\mathbf{K} \mathbf{4}_{\text {core }}^{\square}$, and $\mathbf{S} \mathbf{4}_{\text {core }}^{\square}$ are NL-complete. In the current Section, we present deterministic algorithms for solving these problems. The algorithms will construct a minimal model and check if the formula is satisfied in the root of this model.

The pseudocode for satisfiability checking in $\mathbf{T}_{\text {core }}^{\square}$ is depicted in Algorithm 1. For a given $\mathbf{L}_{\text {core }}^{\square}$-formula $\varphi$ the algorithm constructs a set $M$ of pairs of the form $(w, p)$ such that $(w, p) \in M$ is to mean that in the minimal model $\mathcal{M}_{m d(\varphi), \varphi}^{\mathbf{T}}$ the propositional variable $p$ is satisfied in the world $w$. The construction is initialized in the Lines 1-2 by fixing the size $D$ of the minimal model and adding to $M$ elements corresponding to $V_{0}$ as defined in (3). Afterwards, in the Lines 3-7, the rules $\left(\mathrm{cl}_{1} \mathbf{T}^{\prime}\right)$ and $\left(\mathrm{cl}_{2} \mathbf{T}^{\prime}\right)$ are applied to $M$ until a fixed point is reached (as we have discussed in the previous Section this procedure terminates). It remains to check in the Lines 8-9 whether some clause of the form $\square^{s}\left(p_{1} \wedge p_{2} \rightarrow \perp\right)$ occurring in $\varphi$ raises a contradiction. If this is the case, the input formula is not $\mathbf{T}_{\text {core }}^{\square}$-satisfiable and the algorithm returns 'False', otherwise the formula is $\mathbf{T}_{\text {core }}^{\square}$-satisfiable and the algorithm returns 'True'. 


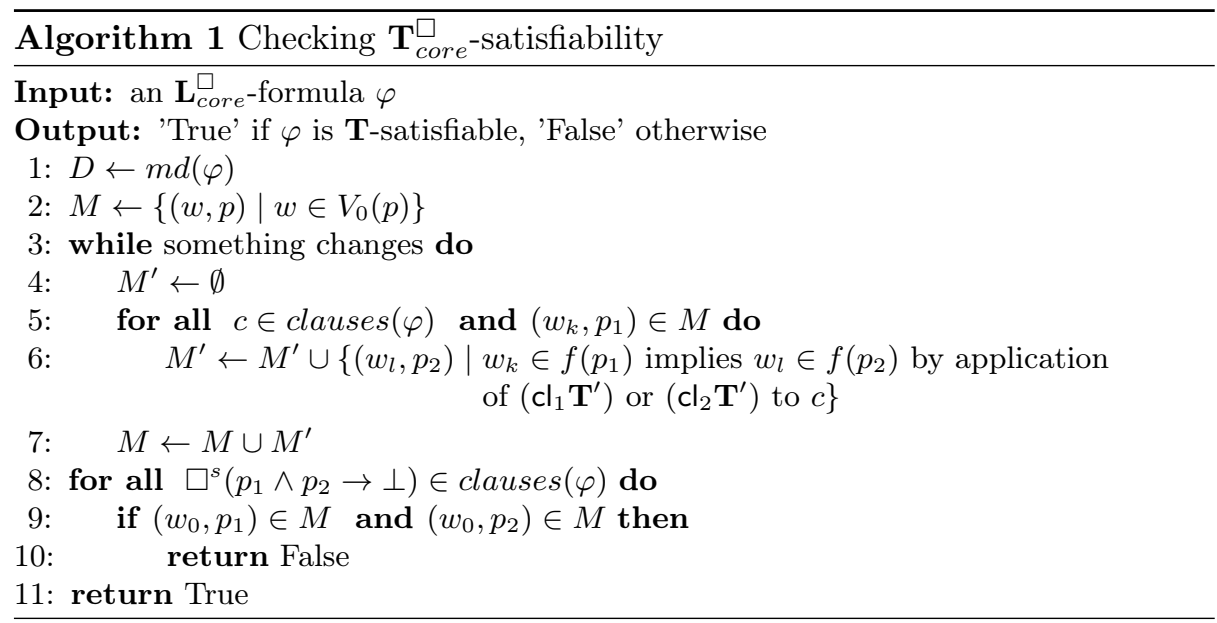

The algorithms for checking $\mathbf{S} \mathbf{4}_{\text {core }}^{\square}$ and $\mathbf{K} \mathbf{4}_{\text {core }}^{\square}$-satisfiability are obtained by suitable modifications of Algorithm 1. The procedure for $\mathbf{S} \mathbf{4}_{\text {core }}^{\square}$-satisfiability requires the following changes: (a) the Line 1 becomes " $D \leftarrow 1$ " as the minimal model for $\mathbf{S} \mathbf{4}_{\text {core }}^{\square}$ is of size 2 , (b) in the Line 6 " $\left(\mathrm{cl}_{1} \mathbf{T}^{\prime}\right)$ or $\left(\mathrm{cl}_{2} \mathbf{T}^{\prime}\right)$ " is replaced with " $\left(\mathrm{cl}_{1} \mathbf{S} 4\right)-\left(\mathrm{cl}_{4} \mathbf{S} 4\right)$ or $\left(\mathrm{cl}_{5} \mathbf{S} \mathbf{4}^{\prime}\right)-\left(\mathrm{cl}_{6} \mathbf{S} \mathbf{4}^{\prime}\right)$ ".

The algorithm for $\mathbf{K} \mathbf{4}_{\text {core }}^{\square}$-satisfiability is more complex. For an $\mathbf{L}_{\text {core }}^{\square}$-formula $\varphi$ it has to be checked for all $D \leq|\varphi|-1$ and for all $x \in\{i, i i\}$ whether $\mathcal{M}_{D, \varphi}^{\mathbf{K} 4(x)}, w_{0} \models \varphi$. Hence, for a fixed $D \leq|\varphi|-1$ and $x \in\{i, i i\}$ the following modifications of Algorithm 1 needs to be done: (a) the Line is 1 deleted because $D$ is already fixed, (b) if $x=i$, then in the Line 6 " $\left(\mathrm{cl}_{1} \mathbf{T}^{\prime}\right)$ or $\left(\mathrm{cl}_{2} \mathbf{T}^{\prime}\right)$ " is replaced with " $\left(\mathrm{cl}_{1} \mathbf{K} 4(i)\right)$ or $\left(\mathrm{cl}_{2} \mathbf{K} 4^{\prime}\right)$ ", otherwise it is replaced with " $\left(\mathrm{cl}_{1} \mathbf{K} 4(i i)\right)$ or $\left(\mathrm{cl}_{2} \mathbf{K} 4^{\prime}\right)$ ", (c) in the Line 9 all occurrences of " $w_{0}$ " are replaced with " $w_{D}$ ".

The above described algorithms construct minimal models of a relevant type and check whether no clause of the form $\square^{s}\left(p_{1} \wedge p_{2} \rightarrow \perp\right)$ raises a contradiction. By Corollaries 6, 10, and 15, these procedures are sufficient for satisfiability checking.

\section{Correspondence to Linear Temporal Logic}

Since there is a close correspondence between the minimal models which we have introduced for checking $\mathbf{T}_{\text {core }}^{\square}, \mathbf{K} \mathbf{4}_{\text {core }}^{\square}$, and $\mathbf{S} \mathbf{4}_{\text {core }}^{\square}$-satisfiability with fragments of the linear temporal logic, our results transfer to the latter.

Let $\bigcirc, \boldsymbol{O}, \square$, and $\boldsymbol{\square}$, be modal temporal operators whose intuitive reading is, respectively, 'in the next time-point', 'now and in the next time-point', 'always in the future', and 'now and always in the future'. For each $X \in\{\bigcirc$ 
let $\mathrm{LTL}_{\text {core }}^{X}$-formulas be generated by the following grammar:

$$
\begin{aligned}
& \varphi_{X}:=\lambda_{X}\left|\neg \lambda_{X}\right| \square^{s}\left(\neg \lambda_{X} \vee \lambda_{X}\right)\left|\square^{s}\left(\neg \lambda_{X} \vee \neg \lambda_{X}\right)\right| \varphi_{X} \wedge \varphi_{X} ; \\
& \lambda_{X}:=\top|p| X \lambda,
\end{aligned}
$$

where $s \in \mathbb{N}$ and $p \in$ PROP.

As usual in linear temporal logic, $\mathrm{LTL}_{\text {core }}^{X}$-formulas are interpreted over a linear order of time-points, namely an LTL-model is a tuple $\mathcal{M}=(T,<, V)$, where $(T,<)$ is a countable discrete and linear order of time-points and the valuation $V:$ PROP $\longrightarrow \mathcal{P}(T)$ assigns sets of time points to propositional variables. The satisfaction relation for a model $\mathcal{M}$ and a time point $t \in T$ is defined for temporal modal operators as follows:

$$
\begin{array}{lll}
\mathcal{M}, t=\bigcirc \varphi & \text { iff } & \mathcal{M}, t^{\prime}=\varphi \text { for } t^{\prime} \text { the immediate }<\text {-succesor of } t ; \\
\mathcal{M}, t=\bigcirc \varphi & \text { iff } & \mathcal{M}, t \models \varphi \wedge \bigcirc \varphi ; \\
\mathcal{M}, t \models \square \varphi & \text { iff } & \mathcal{M}, t^{\prime}=\varphi \text { for all } t^{\prime} \text { such that } t<t^{\prime} ; \\
\mathcal{M}, t \models \square \varphi & \text { iff } & \mathcal{M}, t \models \varphi \wedge \square \varphi,
\end{array}
$$

where $t^{\prime} \in T$ and for other propositional connectives the satisfaction relation is defines as in normal modal logics. Let 0 be the smallest element in $(T,<)$. Then, an LTL-formula $\varphi$ is satisfiable if there exists an LTL-model $\mathcal{M}$ such that $\mathcal{M}, 0=\varphi$.

To show how the fragments of LTL correspond to the fragments of normal modal logics we introduce a translation $\tau_{X}(\varphi)$ for $X \in\{\bigcirc, \boldsymbol{O}, \square, \square\}$ and an $\mathbf{L}_{\text {core }}^{\square}$-formula $\varphi$, such that $\tau_{X}(\varphi)$ is an $\operatorname{LTL}_{\text {core }}^{X}$-formula obtained from $\varphi$ by replacing each occurrence of " $\square$ " in $\varphi$ with " $X$ " except $\square$ 's proceeding clauses, which remain unchanged. Consider the following example:

$$
\begin{aligned}
\varphi & =\square^{2}(p) \wedge \square^{2}(\square p \rightarrow q) ; \\
\tau(\varphi) \bigcirc & =\bigcirc^{2}(p) \wedge \square^{2}(\bigcirc p \rightarrow q) .
\end{aligned}
$$

Then, by the form of minimal models in $\mathbf{K}_{\text {core }}^{\square}, \mathbf{T}_{\text {core }}^{\square}, \mathbf{K} \mathbf{4}_{\text {core }}^{\square}$, and $\mathbf{S} \mathbf{4}_{\text {core }}^{\square}$ we obtain the following correspondence for all $\mathbf{L}_{\text {core }}^{\square}$-formulas $\varphi$ :

$-\varphi$ is $\mathbf{K}_{\text {core }}^{\square}$-satisfiable if an only if $\tau(\varphi) \bigcirc$ is LTL-satisfiable;

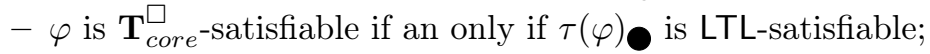

$-\varphi$ is $\mathbf{K} \mathbf{4}_{\text {core }}^{\square}$-satisfiable if an only if $\varphi(\square)$ is LTL-satisfiable;

$-\varphi$ is $\mathbf{S} \mathbf{4}_{\text {core }}^{\square}$-satisfiable if an only if $\tau(\varphi) \mathbf{\square}$ is LTL-satisfiable.

It follows that satisfiability in $\mathrm{LTL}_{\text {core }}^{X}$ is NL-complete for all $X \in\{\bigcirc, \boldsymbol{O}, \square, \mathbf{\square}\}$. A similar result for $L T L_{c o r e}^{\square}$ has been established in [1], where a slightly modified version of the grammar of $L_{T L} L_{c o r e}$-formulas was shown to be NL-complete over time lines coinciding with the standard ordering of integers. The modification was obtained by replacing " $\square$ " preceding clauses in the grammar (4) with the universal modality, stating that a formula holds in all time points. 


\section{Conclusions and Future Work}

In the paper we have showed that the satisfiability problem in core fragments of normal modal logics $\mathbf{T}_{\text {core }}^{\square}, \mathbf{K} \mathbf{4}_{\text {core }}^{\square}$, and $\mathbf{S} \mathbf{4}_{\text {core }}^{\square}$ are NL-complete. We have conducted the proofs by constructing minimal models and checking if the input formula is satisfiable in them. We have showed that for a given $\mathbf{L}_{\text {core }}^{\square}$-formula $\varphi$ there is a single $\mathbf{T}_{\text {core }}^{\square}$ and a single $\mathbf{S} \mathbf{4}_{\text {core }}^{\square}$ minimal model of sizes $\operatorname{md}(\varphi)+1$ and 2, respectively. These results enabled us to construct simple algorithms for satisfiability checking in $\mathbf{T}_{\text {core }}^{\square}$ and $\mathbf{S} \mathbf{4}_{\text {core }}^{\square}$. In the case of $\mathbf{K} \mathbf{4}_{\text {core }}^{\square}$ there are two types of minimal models which make the algorithm more complex.

Moreover, by the form of the minimal models we have showed a correspondence of the above fragments with core fragments of linear temporal logic. Therefore, our computational complexity results for $\mathbf{T}_{\text {core }}^{\square}, \mathbf{K} \mathbf{4}_{\text {core }}^{\square}$, and $\mathbf{S} \mathbf{4}_{\text {core }}^{\square}$ immediately transfer to the corresponding fragments of linear temporal logic.

The complexity map for sub-propositional fragments of normal modal logics still contains a number open problems - see Fig. 1. As a future work we plan to investigate the following questions:

- What is the complexity of $L_{\text {core }}^{\diamond}$ for $L \in\{\mathbf{K} . \mathbf{T}, \mathbf{K} 4, \mathbf{S 4}\}$ ? These fragments are known to be NL-hard and in $\mathrm{P}$ but no tight complexity bounds have been established for them so far;

- What is the computational complexity of core fragments in the basic interval logic, known as the logic of Halpern and Shoham? Is it possible to exploit our proof techniques in the case of interval logics?

Acknowledgments. This work is supported by the National Science Centre in Poland (NCN) grant 2016/23/N/HS1/02168 and by the Foundation for Polish Science (FNP).

\section{References}

1. Artale, A., Kontchakov, R., Ryzhikov, V., Zakharyaschev, M.: The complexity of clausal fragments of LTL. In: International Conference on Logic for Programming Artificial Intelligence and Reasoning. pp. 35-52. Springer (2013)

2. Artale, A., Kontchakov, R., Ryzhikov, V., Zakharyaschev, M.: A cookbook for temporal conceptual data modelling with description logics. ACM Transactions on Computational Logic (TOCL) 15(3), 25 (2014)

3. Blackburn, P., De Rijke, M., Venema, Y.: Modal Logic. Cambridge University Press (2002)

4. Bresolin, D., Kurucz, A., Muñoz-Velasco, E., Ryzhikov, V., Sciavicco, G., Zakharyaschev, M.: Horn fragments of the Halpern-Shoham interval temporal logic. ACM Transactions on Computational Logic (TOCL) 18(3), 22:1-22:39 (2017)

5. Bresolin, D., Muñoz-Velasco, E., Sciavicco, G.: Sub-propositional fragments of the interval temporal logic of Allen's relations. In: European Workshop on Logics in Artificial Intelligence. pp. 122-136. Springer (2014) 
6. Bresolin, D., Munoz-Velasco, E., Sciavicco, G.: On the complexity of fragments of Horn modal logics. In: Temporal Representation and Reasoning (TIME), 2016 23rd International Symposium on. pp. 186-195. IEEE (2016)

7. Halpern, J.Y.: The effect of bounding the number of primitive propositions and the depth of nesting on the complexity of modal logic. Artificial Intelligence 75(2), 361-372 (1995)

8. Halpern, J.Y., Moses, Y.: A guide to completeness and complexity for modal logics of knowledge and belief. Artificial intelligence 54(3), 319-379 (1992)

9. Horn, A.: On sentences which are true of direct unions of algebras. The Journal of Symbolic Logic 16(1), 14-21 (1951)

10. Krom, M.R.: The decision problem for formulas in prenex conjunctive normal form with binary disjunctions. The Journal of Symbolic Logic 35(2), 210-216 (1970)

11. Ladner, R.E.: The computational complexity of provability in systems of modal propositional logic. SIAM Journal on Computing 6(3), 467-480 (1977)

12. Nguyen, L.A.: Constructing the least models for positive modal logic programs. Fundamenta Informaticae 42(1), 29-60 (2000)

13. Nguyen, L.A.: On the complexity of fragments of modal logics. Advances in Modal Logic 5, 318-330 (2004)

14. Papadimitriou, C.H.: Computational Complexity. John Wiley and Sons Ltd. (2003)

15. Sciavicco, G., Muñoz-Velasco, E., Bresolin, D.: On sub-propositional fragments of modal logic. Logical Methods in Computer Science 14 (2018) 